\title{
Article \\ Characteristics of Photon Beam and Preservation of Coherence in Fourth-Generation Light Sources
}

\author{
Bonghoon Oh, Jinjoo Ko *, Jaeyu Lee, Gyeongsu Jang and Seunghwan Shin *
}

check for

updates

Citation: Oh, B.; Ko, J.; Lee, J.; Jang, G.; Shin, S. Characteristics of Photon Beam and Preservation of Coherence in Fourth-Generation Light Sources. Appl. Sci. 2021, 11, 11896. https:// doi.org/10.3390/app112411896

Academic Editor: Edik U. Rafailov

Received: 11 October 2021

Accepted: 6 December 2021

Published: 14 December 2021

Publisher's Note: MDPI stays neutral with regard to jurisdictional claims in published maps and institutional affiliations.

Copyright: (c) 2021 by the authors. Licensee MDPI, Basel, Switzerland. This article is an open access article distributed under the terms and conditions of the Creative Commons Attribution (CC BY) license (https:// creativecommons.org/licenses/by/ $4.0 /)$.
Pohang Accelerator Laboratory, Pohang University of Science and Technology, Pohang 37673, Gyeongbuk, Korea; hope@postech.ac.kr (B.O.); eju@postech.ac.kr (J.L.); jkskid@postech.ac.kr (G.J.)

* Correspondence: kopearl@postech.ac.kr (J.K.); tlssh@postech.ac.kr (S.S.)

Abstract: Fourth-generation storage rings (4GSRs) that exploit the multi-bend achromat lattice concept may be able to surpass the brightness and coherence that are attained using the present third-generation storage rings. This paper presents the characteristics of photon beams and an analysis of their coherence properties in Korea-4GSR to represent 4GSRs.

Keywords: storage ring; multi-bend achromat lattice; brightness; coherence; emittance

\section{Introduction}

The synchrotron radiation (SR) from storage rings has a wide range of applications, including surface science [1], magnetic materials [2], materials chemistry [3], environmental sciences [4], protein crystallography [5], bio-microscopy [6], and chemical dynamics [7]. The SR from storage rings has many notable characteristics including high brilliance and flux, wavelength tunability, beam size tunability, (partially) coherent radiation, polarization, and time structure. In addition, the SR is very stable in energy, intensity, position, and size. The brightness of SR from storage rings has been increased by more than seven orders of magnitude from first-generation to third-generation storage rings (3GSRs; Figure 1). To increase brightness, operation electron beam current has been increased to $400 \mathrm{~mA}$ or higher, and the undulator has been successfully operated to realize a dramatic increase in brightness. At the same time, efforts have been made to increase brightness by reducing electron-beam emittance.
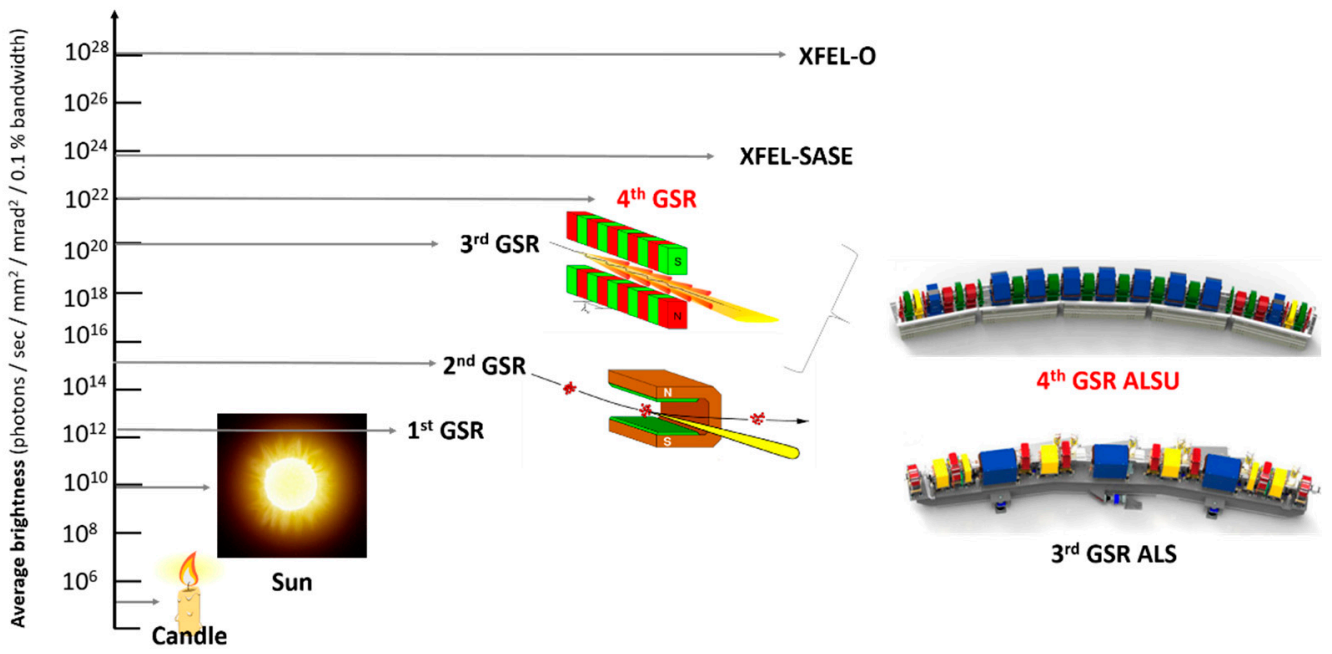

Figure 1. The progress of average brightness. 
The natural emittance in an electron storage ring is given by

$$
\varepsilon_{0}=C_{q} \gamma^{2} \frac{\left\langle H /\left|\rho^{3}\right|\right\rangle}{j_{x}\left\langle 1 / \rho^{2}\right\rangle}
$$

where $C_{q} \cong 3.832 \times 10^{-13} \mathrm{~m}, \gamma$ is the relativistic factor for a particle, $H \cong 1 / \rho^{2}$ (where $\rho$ is the unit-cell bending angle) and $j_{x}$ is the horizontal damping partition number [8-10]. The natural emittance scales with $1 / \rho^{3}$, so the multi-bend achromat lattice concept [11] enables an emittance reduction by one to two orders of magnitude compared with 3GSRs.

The Heisenberg uncertainty principle $\left(\Delta \mathrm{x} \Delta p_{x} \geq \hbar / 2\right)$ sets a lower limit for the emittance of radiation. In storage rings, the altered relation for $S R$ is given by

$$
\Delta x \Delta x^{\prime} \geq \lambda / 4
$$

where $\Delta x, \Delta x^{\prime}$ and $\lambda$ are the position, divergence and wavelength of SR, respectively. If both the horizontal and vertical emittance of the beam are less than $\lambda /(4 \pi)$, then the emitted radiation is fully coherent and the source is diffraction-limited. Here, coherence describes all properties of the correlation between physical quantities of a single wave, or between several waves or wave packets. In a storage ring, coherence is an important parameter in some experiments, such as photon correlation spectroscopy, $\mathrm{X}$-ray holography, and imaging.

Storage rings that exploit the multi-bend achromat (MBA) lattice concept are emerging as part of a worldwide push to move beyond the brightness and coherence reached by present 3GSRs. The first MBA machine was MAX-IV, which began user operations in 2017. SIRIUS [12] and ESRF-EBS [13] are also currently in operation, and several new projects worldwide are exploiting these new concepts, either targeting new light sources such as HEPS, or replacing existing sources; upgrades of APS [14], ALS-U [15], SLS and PETRA-IV are already in progress, and those of DIAMOND, ELETTRA, SOLEIL and others are in a planning phase. The upgraded lattice designs for these facilities have reduced the beam emittance to a few hundred picometers, or even $\leq 100 \mathrm{pm}$. Therefore, 4GSRs can produce coherent beams up to $1 \mathrm{keV}$ X-ray and higher-coherent-fraction X-ray beams $>1 \mathrm{keV}$. In this paper, we address this new era of SR, introduce the parameter of Korea-4GSR to represent 4GSR, and describe the coherent characteristics of SR from 4GSRs.

\section{Worldwide 4GSRs}

The MAX-IV 3-GeV electron storage ring was the first new generation light source that used an MBA lattice [11] to reduce emittance, and hence increase brightness and transverse coherence. Brilliance and coherent fraction are the major performance parameters for 4GSR. Brilliance is defined as the ratio of the photon spectral flux to the phase space volume formed by convolution of the electron and photon beam:

$$
\text { Brilliance }=\frac{f l u x}{4 \pi^{2} \Sigma_{x} \Sigma_{x^{\prime}} \Sigma_{y} \Sigma_{y^{\prime}}}
$$

where the convoluted size is

$$
\Sigma_{x}=\sqrt{\sigma_{x, e}^{2}+\sigma_{p h}^{2}}
$$

and the divergence is

$$
\Sigma_{x^{\prime}}=\sqrt{\sigma_{x^{\prime}, e}^{2}+\sigma_{p h}^{\prime 2}}
$$

where $\sigma_{x, e}$ is electron-beam size, $\sigma_{p h}$ is photon-beam size from a single electron, $\sigma_{x^{\prime}, e}$ is electron-beam divergence, and $\sigma_{p h}^{\prime}$ is photon-beam divergence from a single electron. The 
coherent fraction is represented as the ratio of the photon wavelength to the volume, which is a convolution of the electron and the photon beam in phase space:

$$
F=\frac{\lambda^{2} /(4 \pi)^{2}}{\Sigma_{x} \Sigma_{x^{\prime}} \Sigma_{y} \Sigma_{y^{\prime}}}
$$

where $\lambda$ is the photon wavelength.

Therefore, the brilliance and coherent fraction are maximized by decreasing the electron-beam emittances until the diffraction limit is reached. Typically, an electron emittance of $\sim 10 \mathrm{pm}$ can reach the diffraction limit at $\sim 1 \AA(12.4 \mathrm{keV})$.

Emittance distributions of various storage rings including 4GSRs, tend to decrease as ring circumference increases (Figure 2). The distribution of 3GSR natural emittances indicates that they decrease in inverse proportion to the cube of circumference. By implementing the MBA lattice concept and compact technology, the Korean 4GSR (red asterisk) obtains 100 times the emittance reduction (or even more) of 3GSRs. When an on-axis injection scheme, reverse bends and cutting-edge technology are taken into consideration, emittance is reduced by another $3 \sim 4$ times. Ultra-low emittance rings such as HEPS $(<60 \mathrm{pm})$, Korea-4GSR (58 pm), APS-U (42 pm) and PETRA IV (20 pm) provide high brightness and coherence up to high photon energies.

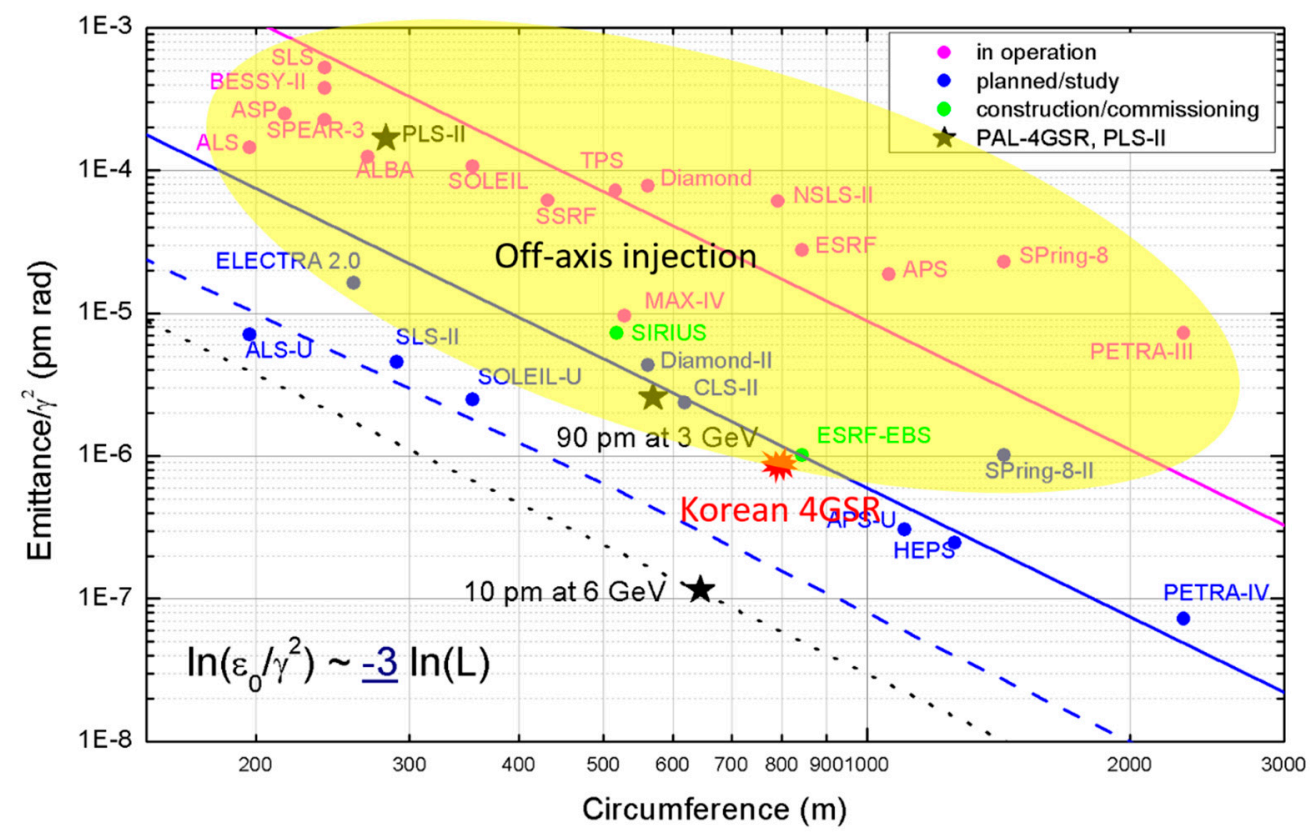

Figure 2. Emittance according to the circumference of 3GSRs and 4GSRs. Vertical axis: natural emittance normalized by the square of energy. Facilities indicated in blue are 4GSRs that use the multi-bend lattice concept.

4GSR projects worldwide (Table 1) choose energies ranging from 2 to $6 \mathrm{GeV}$. All have emittances $<300 \mathrm{pm}$; the lowest is $20 \mathrm{pm}$, which is obtained by PETRA IV, as a consequence of its huge $(2300 \mathrm{~m})$ circumference. The recent trend of 4GSR design is to consider reverse bends. However, not all machines realize beta function optimization for phase-space matching between electron and photon beams. To facilitate beam injection, several machines have a large horizontal beta function at the injection point. 
Table 1. Present ongoing 4GSR projects [16].

\begin{tabular}{|c|c|c|c|c|c|c|}
\hline Project & $\begin{array}{l}\text { Energy } \\
(\mathrm{GeV})\end{array}$ & $\begin{array}{l}\text { Emittance } \\
(\text { pm) }\end{array}$ & $\begin{array}{l}\text { Ener. Spr. } \\
\left(10^{-4}\right)\end{array}$ & $\begin{array}{c}\text { Beta x/y (m) } \\
\text { @ Source Point }\end{array}$ & Reverse Bends & $\begin{array}{l}\text { ID Source } \\
\text { Point Move }\end{array}$ \\
\hline ALS-U & 2.0 & 108 & 9.8 & $2.0 / 2.8$ & yes & No \\
\hline ELETTRA 2 & 2.4 & 212 & 9.3 & $5.7 / 1.6$ & yes & No \\
\hline SLS-II & 2.7 & 157 & 12.0 & $2.5 / 1.3$ & yes & $<70 \mathrm{~mm}$ \\
\hline SOLEIL-U & 2.75 & 81 & 9.0 & $1.3 / 1.3$ & yes & $<100 \mathrm{~mm}$ \\
\hline SIRIUS & 3 & 250 & 8.5 & $1.5 / 1.5$ & no & $\mathrm{n} / \mathrm{a}$ \\
\hline Diamond-II & 3.5 & 136 & 9.0 & $6.0 / 2.5$ & yes & No \\
\hline APS-U & 6 & 42 & 13.5 & $4.9 / 1.9$ & yes & No \\
\hline ESRF-EBS & 6 & 135 & 9.3 & $6.9 / 2.6$ & no & No \\
\hline HEPS & 6 & $<60(35)$ & 10 & $2.6 / 2.3$ & yes & $\mathrm{n} / \mathrm{a}$ \\
\hline PETRA-IV & 6 & 20 & 11.2 & $4.0 / 2.0$ & TBD & yes \\
\hline
\end{tabular}

\section{PLS-II and Korea-4GSR}

This analysis uses parameters (Table 2) of PLS-II [17] to represent 3GSRs, and of Korea-4GSR [18] to represent 4GSRs, to estimate the improvement in coherence in 4GSRs and compare the characteristics of photon beams of 3GSRs to those of 4GSRs. The machine characteristics of PLS-II and Korea-4GSR will be introduced in this section and the results of the investigation of the coherence and characteristics of photon beams will be described in the next section.

Table 2. Major parameters of the Korea-4GSR storage ring.

\begin{tabular}{cccc}
\hline Parameter & PLS-II & Korea-4GSR & Unit \\
\hline Beam energy & 3 & 4 & $\mathrm{GeV}$ \\
Beam current & 400 & 400 & $\mathrm{~mA}$ \\
Lattice structure & $\mathrm{DBA}$ & Hybrid 7BA & \\
Superperiods & 12 & 28 & \\
Natural hor. emittance & 5.8 & 0.058 & $\mathrm{~nm} \mathrm{rad}$ \\
Betatron tune & $15.28 / 9.18$ & $67.44 / 23.17$ & $\mathrm{MHz}$ \\
RF frequency & 500 & 500 & $\%$ \\
rms energy spread & 0.1 & 0.1 & $\mathrm{ps}$ \\
rms bunch length & 20 & 13 & \\
\hline
\end{tabular}

The PLS-II lattice has a gradient dipole double-bend achromat structure with 12 superperiods. Each of the 12 super-periods contains two gradient dipole magnets equipped with a FODO cell, which is a lattice period composed of a focusing (F) and a defocusing (D) quadrupole with a drift space $(\mathrm{O})$ arrangement. The critical photon energy from bending magnets is $8.97 \mathrm{keV}$. The lattice provides a $6.88 \mathrm{~m}$ and a $3.69 \mathrm{~m}$ straight section per cell for installation of insertion devices. To reduce the beam emittance, a horizontal dispersion of $0.25 \mathrm{~m}$ is considered in the long straight section, in which RF cavities are installed. The lattice functions (Figure 3) for the PLS-II illustrate how a compact lattice can be realized to accommodate twenty insertion devices along the $280 \mathrm{~m}$ circumference. 


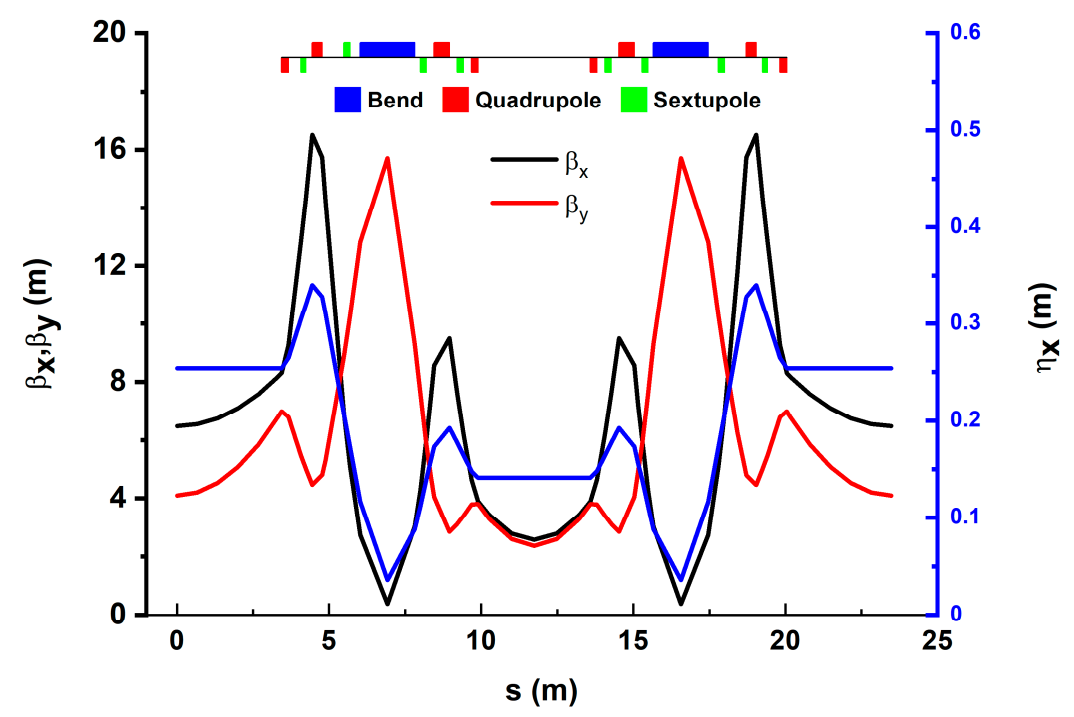

Figure 3. Super-period in a designed lattice for the $3.0 \mathrm{GeV}$ ring of PLS-II and its lattice function.

The Korea-4GSR lattice has a hybrid seven-bend achromat (H7BA) type with a $58 \mathrm{pm}$ horizontal emittance. The ring with an $800 \mathrm{~m}$ circumference is composed of 28 symmetric cells. As a result of experience with the PLS-II, the length of the straight section in the Korea 4GSR is set to $6.5 \mathrm{~m}$ to accommodate two SCRF modules. The Korea-4GSR lattice has a 2-T high field central dipole to create radiation that has a critical energy of $21 \mathrm{keV}$. The Korea-4GSR uses ESRF-EBS and APS-U lattices. The dispersion was deliberately magnified between the first and second dipoles and between the sixth and seventh dipoles to make a dispersion bump. Chromatic sextupoles were located in this bump region to control the chromaticity with smaller strength. The betatron phase advances between the two dispersion bumps were adjusted to be $\Delta \varphi_{\mathrm{x}} \sim 3 \pi$ in the horizontal plane and $\Delta \varphi_{\mathrm{y}} \sim \pi$ in the vertical plane. Consequently, nonchromatic effects generated by the sextupoles are canceled out naturally. Five-step longitudinal gradient bends and reverse dipoles were introduced to further reduce natural emittance. Each super-period in the designed lattice for the Korea-4GSR is composed of a total of 28 cells (Figure 4).

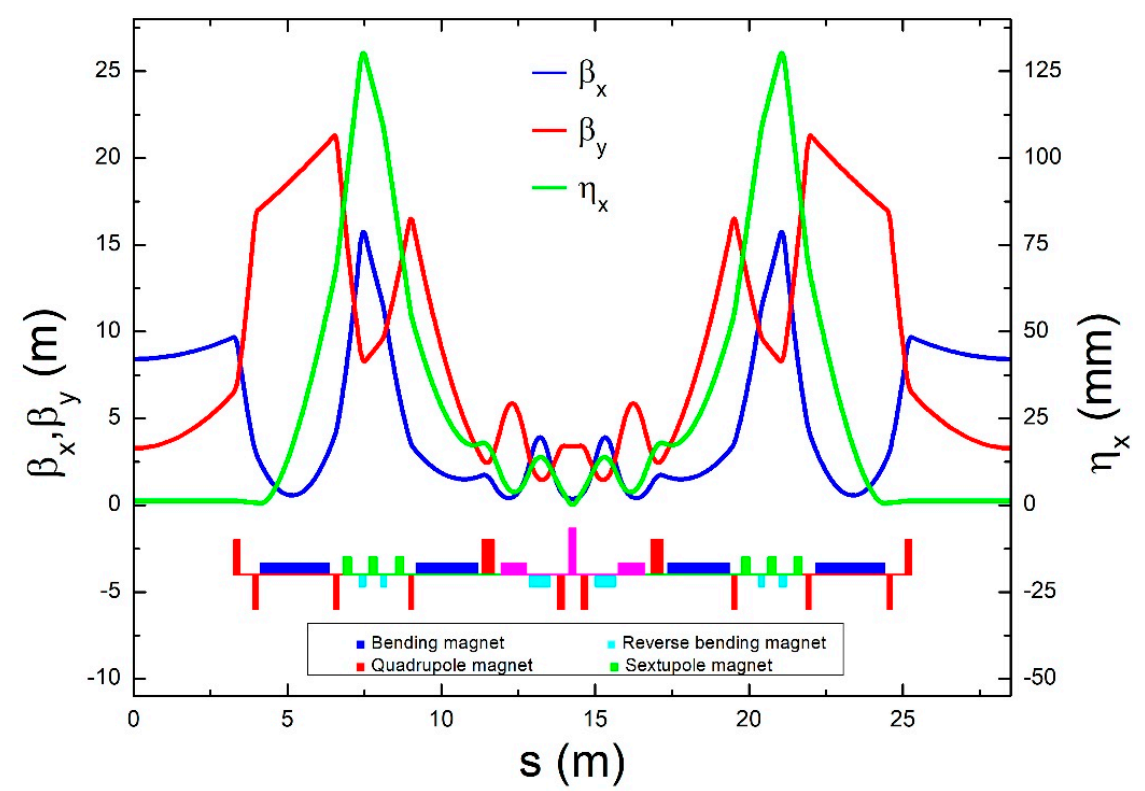

Figure 4. Super-period in a designed lattice for the $4.0 \mathrm{GeV}$ ring of Korea-4GSR and its lattice function. 
The Korea-4GSR project has officially been started. The conceptual design project for Korea-4GSR is complete, and the overall project will be completed in 2027. It will have extremely low emittance (Figure 2) and a conventional off-axis injection scheme is being considered. Korea-4GSR has 1/100 the emittance of PLS-II as a result of using the multi-bend lattice concept (Table 2).

\section{Characteristics of Photon Beams}

The quality of SR can be evaluated by many variables, of which flux, brilliance, and transverse coherence are important. To compare three important characteristics of PLS-II and Korea-4GSR, three representative insertion devices, which have already been operated in PLS-II, are considered with two different bending magnets to evaluate photon beam characteristics (Table 3).

Table 3. Major parameters of three representative insertion device in PLS-II.

\begin{tabular}{cccc}
\hline Parameter & Wiggler & EPU & In-Vac. Undulator \\
\hline Total length $(\mathrm{m})$ & 2.0 & 3.6 & 1.3 \\
Peak field $(\mathrm{T})$ & 1.81 & 0.89 & 0.97 \\
Peroid of ID $(\mathrm{cm})$ & 10 & 11.4 & 2 \\
\hline
\end{tabular}

The total flux of a synchrotron light source is defined as the total number of photons emitted per unit of time at a given spectral bandwidth. Therefore, the total flux is independent of beam emittance and strongly depends on the current and energy of the electron beam. Compared to the PLS-II, the flux curve for Korea-4GSR is shifted to the right due to an electron beam energy increase (Figure $5 a$ ).

(a)

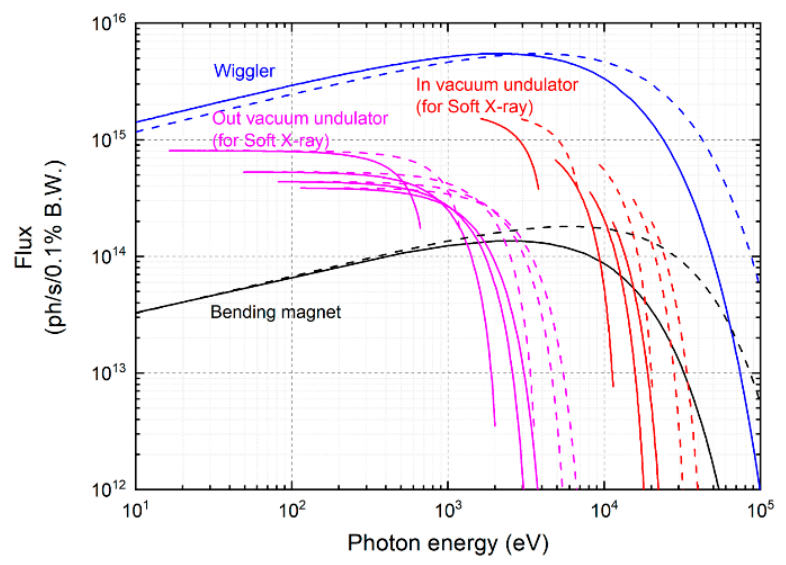

(b)

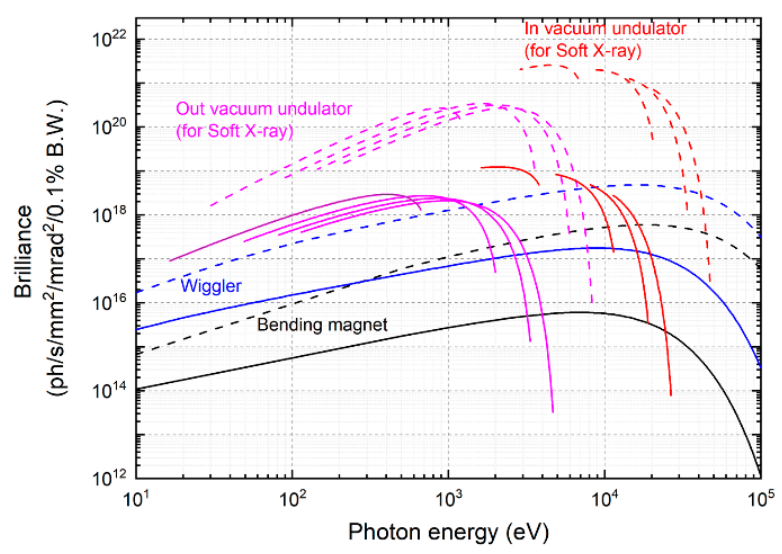

Figure 5. Comparison of (a) photon beam flux and (b) brilliance of Korea-4GSR with PLS-II for different radiation sources. For each color, the solid line indicates PLS-II, and the dotted line indicates Korea-4GSR. Bending magnet (black), wiggler (blue), out-vacuum undulator (pink) and in-vacuum undulator (red).

The brilliance is defined as the total number of photons emitted per unit of time, and per unit of area in the transverse direction at a given spectral bandwidth. In the case of bending magnets, the horizontal aperture was set to $5 \mathrm{mrad}$ for PLS-II and Korea4GSR. At $10 \mathrm{keV}$, Korea-4GSR is 100 times higher than PLS-II, because the emittance of the Korea-4GSR is reduced (Figure $5 b$ ).

The photon-density distributions in phase space were obtained (Figure 6) for the bending magnet, wiggler, and out-vacuum undulator and in-vacuum undulator in PLSII and Korea-4GSR. The increased density of distribution (i.e., improvement) of Korea4GSR was particularly extreme in beam size and divergence in the horizontal plane. This improvement is a result of the smaller electron emittance and zero dispersion in straight sections in Korea-4GSR than in PLS-II. The wiggler radiation assumes a toroid shape in the 
phase-space photon beam density of Korea-4GSR, because wiggling motion is dominated by electron beam emittance reduction (Figure 6b). This specific shape in phase space should be included in the beamline design. In addition, the vertical photon-density distribution of the soft X-ray region of both PLS-II and Korea-4GSR represents a diffraction-limit pattern close to the SR pattern emitted from single electron (Figure 6o,p).
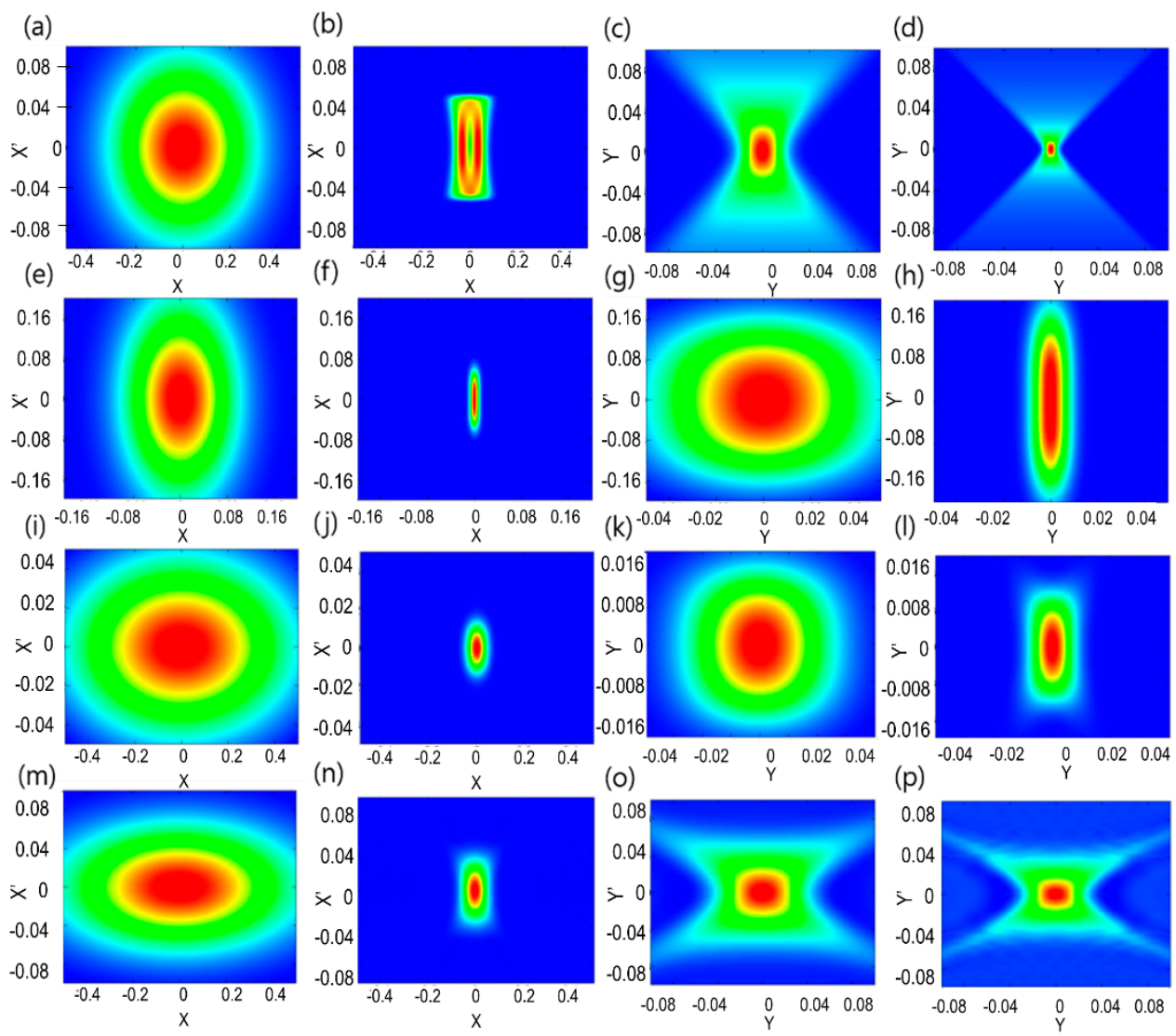

Figure 6. Phase space photon beam distribution from four different types of light source. Horizontal phase space @ 10-keV photon beam from (a) wiggler in PLS-II, (b) wiggler in Korea-4GSR. Vertical phase space @ 10-keV photon beam from (c) wiggler in PLS-II, (d) wiggler in Korea-4GSR. Horizontal phase space @ 10-keV photon beam from (e) bending magnet in PLS-II, (f) bending magnet in Korea4GSR. Vertical phase space @ 10-keV photon beam from $(\mathrm{g})$ bending magnet in PLS-II, (h) bending magnet in Korea-4GSR. Horizontal phase space @ 8-keV photon beam from (i) in-vacuum undulator in PLS-II, (j) in-vacuum undulator in Korea-4GSR. Vertical phase space @ 8-keV photon beam from (k) in-vacuum undulator in PLS-II, (1) in-vacuum undulator in Korea-4GSR. Horizontal phase space @ 0.2-keV photon beam from (m) out-vacuum undulator in PLS-II, (n) out-vacuum undulator in Korea-4GSR. Vertical phase space @ 0.2-keV photon beam from (o) out-vacuum undulator in PLS-II, (p) out-vacuum undulator in Korea-4GSR.

\section{Preservation of Coherence}

As emittance approaches the diffraction limit, the coherence of SR increases in the transverse dimension. To quantify the degree of coherence, the fraction of coherence, defined in Equation (6) was evaluated (Figure 7) to be about 100 times higher, on average, in Korea-4GSR than in PLS-II. 


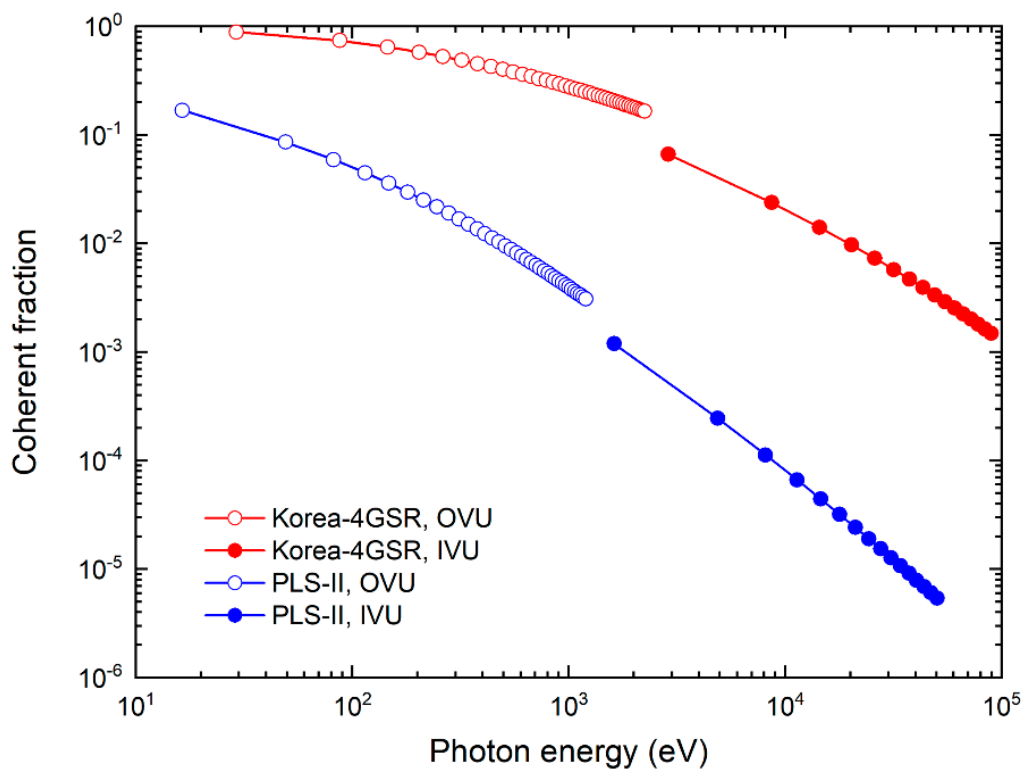

Figure 7. Comparison of the transverse coherent fraction between Korea-4GSR and PLS-II. An out-vacuum undulator (OVU) is applied below about $1 \mathrm{keV}$, and an in-vacuum undulator (IVU) is applied above about $2 \mathrm{keV}$.

To evaluate the coherence fraction along a wide spectrum range, an out-vacuum undulator and an in-vacuum undulator were considered as SR sources. The out-vacuum undulator generates $\mathrm{SR}$ up to $1 \sim 3 \mathrm{keV}$, and the in-vacuum undulator generates $\mathrm{SR}>1 \mathrm{keV}$ (Figure 8). The coherence fraction beyond $10 \mathrm{keV}$ is at most a few percent coherent. To satisfy high coherency at $10 \mathrm{keV}$, electron emittance $<10 \mathrm{pm}$ should be realized. However, currently, the storage ring yields a partially coherent beam at $10 \mathrm{keV}$.

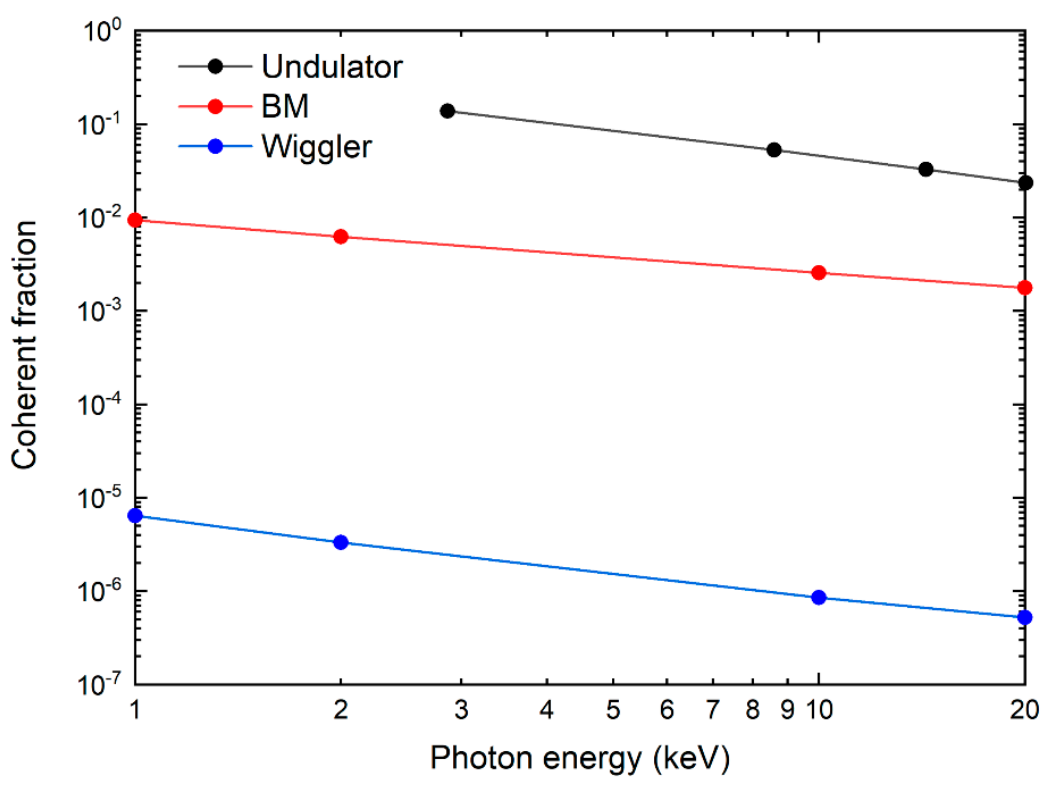

Figure 8. Comparison of the transverse coherent fraction of Korea-4GSR among undulator, bending magnet (2T central dipole), and wiggler.

Coherence also depends on the SR source, as well as on the lattice function at the source point. The undulator is the strongest coherent SR source due to the small electronbeam path variation in the SR region. A comparison of coherence among the undulator, bending magnet, and wiggler at the given parameter of the storage ring demonstrates that an undulator must be used for experiments that require high coherence (Figure 8). 
The improvements in photon beam coherence caused by a reduction in electron beam emittance will be amplified by matching the phase-space distributions of the electron beam and photon beam. Proper matching of the phase space distribution of the electron beam to the photon beam requires tuning of the betatron function. The optimal betatron function that provides maximum coherence and brilliance is [19-21].

$$
\beta_{x, y}^{o p t} \sim L / \pi
$$

where $L$ is the undulator length. To investigate the optimal conditions of each SR source for Korea-4GSR, the coherences and brilliances of each SR source were quantified as a function of the betatron function. At optimal conditions along the betatron function, the coherence of the undulator is increased to $30 \%$ by reducing the betatron function to $1 \mathrm{~m}$ (Figure 9). Coherence in the wiggler source does not depend on the betatron function, because coherence is dominantly determined by large electron-beam path variation rather than by phase-matching in the wiggler source. The coherence of the photon beam from a bending magnet is strongly dependent on the betatron function, and less dependent on the undulator.

$$
\text { (a) }
$$
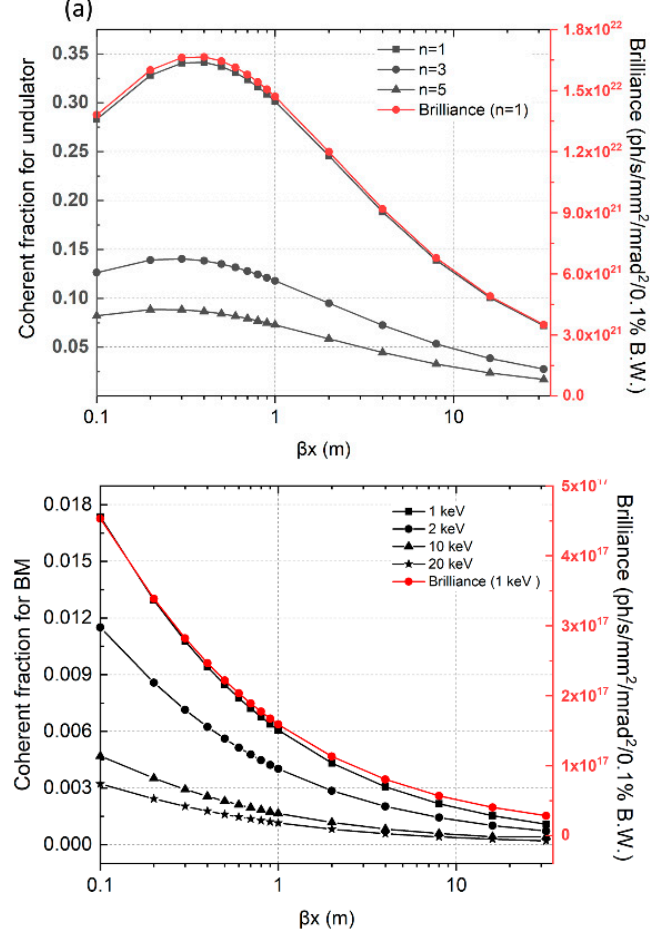

(b)

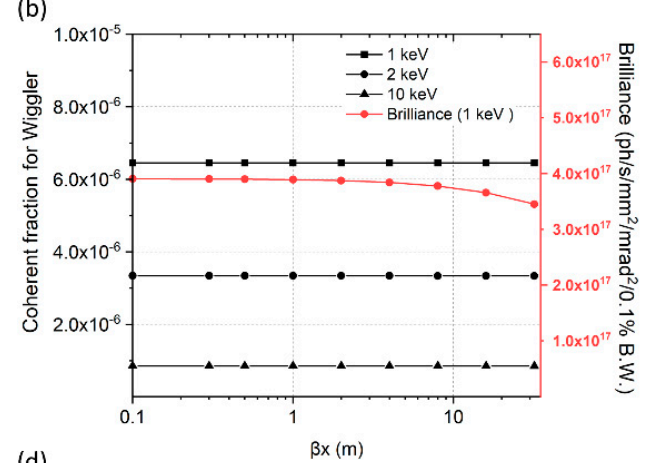

(d)

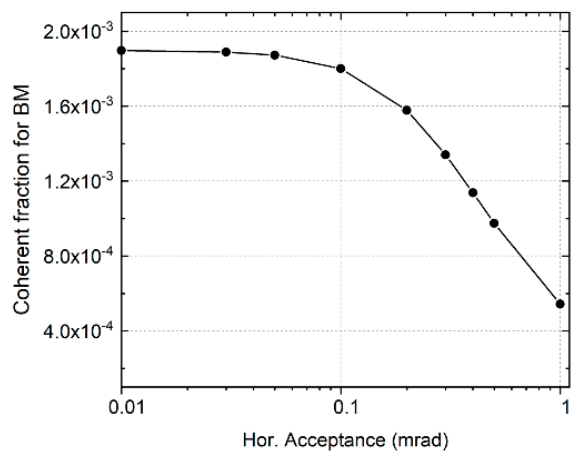

Figure 9. Horizontal coherent fraction of Korea-4GSR as a function of the betatron function for (a) undulator, (b) wiggler, and (c) bending magnet. (d) Horizontal coherent fraction of the bending magnet as a function of the horizontal acceptance.

The effect of error of focusing by the mirror was quantified to identify the tolerance range of such errors. The errors of the focusing mirror are caused by vibration of the mirror (slop error) and error of the mirror surface (roughness). These errors are a direct cause of deterioration in the coherence of the beam. For example, given a focusing mirror that is at a distance $p$ from the SR source, then, if the mirror receives the full beam, the beam spread is approximately $2 p \sigma_{v i b}$ due to the vibration $\sigma_{v i b}$, or $2 p \sigma_{\text {tilt }}$ due to the tilt error of the mirror $\sigma_{\text {tilt }}$. In addition, the coherence characteristics of the photon beam decline and can be defined as [22]

$$
\frac{F_{c} \Sigma_{x}}{\sqrt{\Sigma_{x}^{2}+\Delta \sigma_{x}^{2}}} \text { or } \frac{F_{c} \Sigma_{y}}{\sqrt{\Sigma_{y}^{2}+\Delta \sigma_{y}^{2}}},
$$


where $F_{c}$ is the coherent fraction when the mirror is error-free, $x$ is horizontal direction, $y$ is vertical direction, and $\sigma$ represents an error due to the vibration or tilt of the mirror. Equation (8) allows for a comparison of the error effects on coherent flux in PLS-II and Korea-4GSR (Figure 10). For the same error, the deterioration of coherent flux is severe in the horizontal plane in PLS-II, and in the vertical plane in Korea-4GSR. Therefore, these results indicate the need for a thorough study of the effect of error on beam coherence during the design of a Korea-4GSR beamline.

(a)

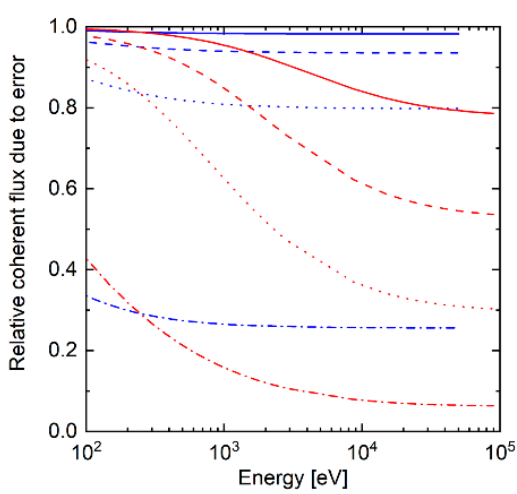

(b)

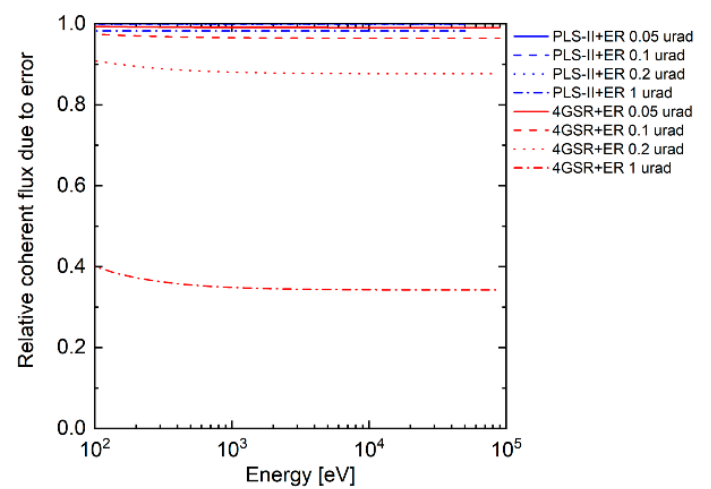

Figure 10. Comparison of error effect on coherent flux between the PLS-II and Korea-4GSR in (a) vertical and (b) horizontal directions. ER in legend means $\sigma_{v i b}$, or $\sigma_{\text {tilt }}$.

\section{Conclusions}

Fourth-generation storage rings are being developed. They are expected to increase the coherence of the SR beam by reducing beam emittance to a few hundred picometers, or even $<100 \mathrm{pm}$. In this paper, we described a quantitative estimation of the coherence of photon beams by using machine parameters from PLS-II as an example of a 3GSR, and Korea-4GSR as an example of a 4GSR. An undulator should be used as the SR source with the optimized betatron function to increase the coherence of the photon beam. With optimized conditions, Korea-4GSR will provide coherence beams with a $30 \%$ coherent fraction at $10-\mathrm{keV}$ photon-beam energy, which is 100 times higher, on average, than that of PLS-II. The need for a thorough study of error effect on coherence beam when designing the Korea-4GSR beamline was also suggested, because errors in optic elements in Korea-4GSR degrade coherent flux.

Author Contributions: Conceptualization, S.S. and B.O.; methodology, S.S.; software, J.K.; validation, G.J.; formal analysis, J.K.; investigation, J.L.; resources, J.L.; data curation, J.L.; writing—original draft preparation, S.S.; writing—review and editing, B.O.; visualization, G.J.; supervision, S.S.; project administration, S.S.; funding acquisition, S.S. All authors have read and agreed to the published version of the manuscript.

Funding: This research received no external funding.

Institutional Review Board Statement: Not applicable.

Informed Consent Statement: Not applicable.

Data Availability Statement: Not applicable.

Acknowledgments: This research was supported by the Basic Science Research Program through the National Research Foundation of Korea (NRF-2019R1C1C1003412) and by the Basic Science Research Program through the National Research Foundation of Korea (NRF-2019R1C2C1004862).

Conflicts of Interest: The authors declare no conflict of interest. 


\section{References}

1. Panaccione, G. Advanced photoelectric effect experiment beamline at Elettra: A surface science laboratory coupled with Synchrotron Radiation. Rev. Sci. Instrum. 2009, 80, 043105. [CrossRef] [PubMed]

2. Kortright, J.; Awschalom, D.; Stöhr, J.; Bader, S.; Idzerda, Y.; Parkin, S.; Schuller, I.K.; Siegmann, H.-C. Research frontiers in magnetic materials at soft X-ray synchrotron radiation facilities. J. Magn. Magn. Mater. 1999, 207, 7-44. [CrossRef]

3. Saisho, H.; Gohshi, Y. Applications of Synchrotron Radiation to Materials Analysis; Elsevier: Amsterdam, The Netherlands, 1996.

4. Brown, E.; Sturchio, C. An Overview of Synchrotron Radiation Applications to Low Temperature Geochemistry and Environmental Science. Rev. Mineral. Geochem. 2002, 49, 1-115. [CrossRef]

5. Helliwell, J.R. Macromolecular Crystallography with Synchrotron Radiation; Cambridge University Press: Cambridge, UK, 1992.

6. Tang, R. Microbubble-based synchrotron radiation phase contrast imaging: Basic study and angiography applications. Phys. Med. Biol. 2011, 56, 3503. [CrossRef] [PubMed]

7. Sham, T.-K. Chemical Applications of Synchrotron Radiation; World Scientific: Singapore, 2002.

8. Sands, M. SLAC-121.Stanford Linear Accelerator Center. 1970. Available online: https://digital.library.unt.edu/ark:/67531 / metadc865991/m1/ (accessed on 10 October 2021).

9. Wiedemann, H. Particle Accelerator Physics I; Springer: Berlin/Heidelberg, Germany, 1993.

10. Borland, M.J. Lattice design challenges for fourth-generation storage-ring light sources. Synchrotron Radiat. 2014, $21,912$. [CrossRef] [PubMed]

11. Einfeld, D.; Plesko, M.; Schaper, J. First multi-bend achromat lattice consideration. Synchrotron Radiat. 2014, 21, 856. [CrossRef] [PubMed]

12. Liu, L.; Milas, N.; Mukai, A.H.C.; Resende, X.R.; Rodriguet, A.R.D.; Sa, F.H. A New 5BA Low Emittance Lattice for Sirius. In Proceedings of the 4th International Particle Accelerator Conference, Shanghai, China, 12-17 May 2013; p. 1874.

13. ESRF-EBS Introduction. Available online: http:/ /indico.psi.ch/conferenceDisplay.py?confId=5589 (accessed on 10 October 2021).

14. APS Upgrade Introduction. Available online: https:// www.aps.anl.gov/APSUpgrade (accessed on 10 October 2021).

15. Steier, C. Physics design progress towards a diffraction limited upgrade of the ALS. In Proceedings of the 7th International Particle Accelerator Conference IPAC 2016, Busan, Korea, 8-13 May 2016; p. 2956.

16. Bartolini, R. Overview of Ongoing Fourth-Generation Light Source Projects Worldwide. Available online: https://www.maxiv.lu. se/news/7th-dlsr-2021/ (accessed on 10 October 2021).

17. Shin, S.; Kwon, S.; Kim, D.T.; Kim, D.E.; Kim, M.; Kim, S.H.; Kim, S.C.; Kim, J.; Kim, C.; Park, B.; et al. Commissioning of the PLS-II. J. Instrum. 2013, 8, 01019. [CrossRef]

18. Lee, J.; Jang, G.; Ko, J.; Shin, S. Storage Rings in Korea as Synchrotron Radiation Source. J. Korean Phys. Society. 2021. submitted.

19. Liu, L.; Westfahl, H., Jr. Toward Diffraction Limited Storage Ring Based Light Sources. In Proceedings of the 8th International Particle Accelerator Conference, Copenhagen, Denmark, 14-19 May 2017.

20. Hettel, R. DLSR design and plans: An international overview. J. Synchrotron Radiat. 2014, 21, 843-855. [CrossRef] [PubMed]

21. Sun, C.; Robin, D.S.; Nishimura, H.; Steier, C.; Wan, W. Small-emittance and low-beta lattice designs and optimizations. Phys. Rev. Spec. Top. Accel. Beams 2012, 15, 054001. [CrossRef]

22. Shi, X. APS-U Emittance Studies-Optics Consideration. Available online: https://www.aps.anl.gov/files/APS-Uploads/ApsUpgrade/APS-U\%20Forum\%20Jan_14_2016\%20Xianbo\%20Shi.pdf (accessed on 14 January 2015). 